\section{Perfil de morbidade e de mortalidade de pacientes idosos hospitalizados}

\author{
Morbidity and mortality profile \\ of hospitalized elderly patients
}

\author{
Ana Claudia Santos Amaral 1 \\ Cláudia Medina Coeli ${ }^{1}$ \\ Maria do Carmo Esteves da Costa 2 \\ Vânia da Silva Cardoso 1 \\ Ana Lúcia Araújo de Toledo 1 \\ Carla Rodrigues Fernandes 1
}

1 Núcleo de Estudos de Saúde
Coletiva, Universidade
Federal do Rio de Janeiro,
Rio de Janeiro, Brasil.
2 Secretaria Municipal de
Saúde do Rio de Janeiro,
Rio de Janeiro, Brasil.
Correspondência
A. C. S. Amaral
Núcleo de Estudos de Saúde
Coletiva, Universidade
Federal do Rio de Janeiro.
Av. Brigadeiro Trompowsky
s/no, Hospital Universitário
Clementino Fraga Filho,
5o andar, Ala Sul,
Cidade Universitária,
Rio de Janeiro-RJ
21941-590, Brasil.
acsamaral@ig.com.br

Abstract

The objectives of this study were to analyze the morbidity and mortality profile in elderly $\mathrm{pa}$ tients hospitalized in two teaching and two non-teaching hospitals in Rio de Janeiro, Brazil (Municipal Planning Area 2.2) in 1999, and to compare in-hospital mortality rates adjusted for differences in profile. Data were obtained from the National Hospital Database of the Unified National Health System (SIHISUS). The logistic model included the variables age and primary diagnosis to calculate risk-adjusted inhospital mortality rates. Hospital admissions of elderly patients $(n=7,584)$ represented $29.3 \%$ of a total of 25,928 hospitalizations that took place in these units. Senile cataract (7.8\%) was the most frequent cause of hospitalization, followed by prostate hyperplasia (4.7\%), congestive heart failure (2.9\%), and complete atrioventricular block (2.8\%). Non-teaching hospitals presented in-hospital mortality rates higher than teaching hospitals even after adjusting for case profile differences with regard to age and primary diagnosis. The use of SIH/SUS databases and the risk adjustment methodology represent an alternative for exploratory analysis of healthcare outcomes.

Aging Health; Hospitalization; Hospital Mortality; Morbidity

\section{Introdução}

Os países do chamado Terceiro Mundo vêm apresentando, nas últimas décadas, um progressivo declínio nas suas taxas de mortalidade e, mais recentemente, também nas suas taxas de fecundidade. Esses dois fatores associados promovem a base demográfica para um envelhecimento real dessas populações, à semelhança do processo que continua ocorrendo, ainda que em escala menos acentuada, nos países desenvolvidos 1 .

A passagem de uma situação de alta mortalidade e alta fecundidade para uma de baixa mortalidade e, gradualmente, baixa fecundidade, como a que se observa atualmente no Brasil, traduz-se numa elevação da expectativa de vida média da população e num aumento em termos absolutos e proporcionais do número de pessoas atingindo idades avançadas 1. Projeções recém-publicadas pela Organização Mundial da Saúde estimam que, entre 1990 e 2025, a população idosa aumentará de sete a oito vezes em países como Colômbia, Malásia, Quênia, Tailândia e Gana. As mesmas projeções indicam que entre os dez países com as maiores populações idosas em 2025, cinco serão países em desenvolvimento, incluindo o Brasil com um número estimado de 27 milhões de pessoas com sessenta anos ou mais de idade 2 .

A problemática decorrente do envelhecimento, no que diz respeito à saúde, tende a ser 
a mesma que se verifica nos países desenvolvidos (doenças crônicas requerendo cuidados continuados e custosos), agravada pelo fato de persistirem enquanto prioridades problemas como desnutrição e doenças infecciosas 1 . Os idosos tendem a consumir mais serviços de saúde, apresentando taxas de internação hospitalar bem mais elevadas do que as observadas em outros grupos etários, assim como uma permanência hospitalar mais prolongada 3,4 .

$\mathrm{O}$ investimento na saúde e educação da atual população de jovens e a redução da morbidade são apresentadas como alternativas capazes de minimizar, em um país com recursos financeiros escassos, o impacto do envelhecimento populacional sobre a qualidade de vida 5,6 , porém o custo elevado da assistência à saúde na terceira idade, de três a sete vezes mais caro que o custo médio da população, tem inibido as iniciativas do poder público dirigidas a essa faixa etária 7 .

Diante disso, ênfase deve ser dada à avaliação dos cuidados de saúde prestados a este seguimento populacional, incluindo aqueles proporcionados pelos hospitais, tanto para promover maior conhecimento sobre a efetividade desses cuidados, quanto para proporcionar maior eficiência na utilização de recursos de saúde. A realização de avaliações sobre cuidados de saúde implica a necessidade da utilização de medidas válidas e eficientes que permitam a realização de comparações de desempenho entre os hospitais 8,9.

Os bancos de dados, desenhados para fins administrativos, que contêm as notas de reembolso hospitalar expandiram-se e passaram a incluir informações clínicas, possibilitando seu uso para a avaliação dos cuidados prestados aos pacientes 10. Estas bases de dados vêm sendo empregadas com o objetivo de gerar indicadores de resultados que representem um ponto de partida para avaliações mais detalhadas da qualidade da assistência hospitalar 11 .

No Brasil, o Sistema de Informações Hospitalares do Sistema Único de Saúde (SIH/SUS) é um exemplo de um banco de dados desenhado para fins administrativos, usado por meio de um sistema que é responsável pelo processamento dos registros contidos nos formulários de Autorização de Internação Hospitalar (AIH), oferecendo grandes vantagens para avaliação de qualidade por conterem grande volume de dados, disponíveis com grande proximidade da ocorrência da internação, reduzindo em muito o custo das avaliações 8 .

O presente estudo tem por objetivos estudar o perfil de morbi-mortalidade de idosos hospitalizados em unidades públicas da Área
Programática 2.2 (AP2.2), da cidade do Rio de Janeiro, Brasil, no ano de 1999, tendo como base os dados do SIH/SUS, e avaliar comparativamente a mortalidade hospitalar nestas unidades empregando medidas de ajuste para diferenças no perfil dos pacientes hospitalizados.

\section{Metodologia}

\section{População de estudo}

Foram analisadas as internações ocorridas em pacientes com sessenta anos ou mais de idade, no ano de 1999, em quatro unidades hospitalares localizadas na AP2.2. Esta área representa uma subdivisão do Município do Rio de Janeiro que engloba regiões administrativas e bairros, que por suas vocações e peculiaridades do uso do solo, a prefeitura da cidade utiliza para o planejamento e implementação de políticas públicas.

Os hospitais analisados possuem características bem distintas, dentre eles: um hospital municipalizado, com atendimento de emergência e capacidade instalada de 300 leitos (Unidade 1); um hospital universitário (Unidade 2), de atendimento geral sem emergência, com capacidade instalada de 198 leitos; um hospital universitário apresentando o mesmo perfil de atendimento com capacidade de 601 leitos (Unidade 3); e por último, um hospital municipal (Unidade 4), com atendimento especificamente geriátrico e capacidade instalada de 26 leitos (a unidade ainda mantém 32 leitos de gerontologia não cadastrados no SIH/ SUS) (http://www.cnes.datasus.gov.br/Mod. Perguntas6.asp, acessado em 01/Ago/2001).

\section{Fonte de dados}

Foram analisadas as AIH que constituem um banco de dados informatizado, processado nacionalmente pelo SIH/SUS. A AIH é o instrumento de informações e cobranças dos serviços prestados aos usuários do SUS. A unidade de identificação das internações é um número seqüencial que individualiza cada formulário AIH 8,12. Para análise deste trabalho foram utilizadas apenas as AIH tipo 1, aquelas emitidas no início da internação pelo SUS, bem como, consideradas apenas as internações de pacientes maiores de sessenta anos de idade. Os dados utilizados foram extraídos de arquivos compactados, armazenados em CD-ROM do DATASUS, no ano de 1999 (http://www.datasus.gov.br/arquivos/ftpdata.htm\#dados, acessado em 01/Ago/2001). 


\section{Análise de dados}

As variáveis estudadas foram: sexo; faixa etária (intervalos de cinco anos); especialidade da internação; diagnóstico primário, agregando as categorias e subcategorias dos capítulos da 10a revisão da Classificação Estatística Internacional de Doenças e Problemas Relacionados à Saúde (CID-10) 13; e tempo de permanência.

Para cada unidade de saúde foram estimadas as taxas de mortalidade hospitalar brutas e seus respectivos intervalos de confiança de $95 \%$, calculados por meio do método exato. Para a estimativa das taxas de mortalidade hospitalar ajustadas foi inicialmente construído um modelo de regressão logística para o conjunto das hospitalizações, incluindo as seguintes variáveis: sexo, idade (contínua) e capítulo da CID-10 relativo ao diagnóstico principal. Os capítulos com freqüência de ocorrência inferior a 1,0\% ou que não apresentaram ao menos um caso de óbito foram agrupados ficando a distribuição final igual a I, II, IV, VI, IX, X, XI, XII, XIII, XIV, XVIII, XIX e demais capítulos. A variável sexo não apresentou associação significativa com o óbito e foi eliminada, desta forma o modelo final incluiu apenas as variáveis idade e capítulos da CID-10. A calibração do modelo final foi avaliada pelo teste de HosmerLemeshow e o poder de discriminação foi avaliado pela área sob a curva ROC (estatística c) 14 . $\mathrm{O}$ modelo apresentou um bom ajuste aos da$\operatorname{dos}(\mathrm{H}-\mathrm{Lc} 2$ [gl 8] = 8.11; $\mathrm{p}=0,42)$ e uma boa performance preditiva $(c=0,75)$. Este modelo foi, então, empregado para a estimativa de óbitos esperados em cada unidade de saúde. A seguir foi calculada para cada hospital a razão de óbitos observados em relação aos óbitos esperados. Por fim, a taxa de mortalidade ajustada foi calculada usando-se a multiplicação da razão entre óbitos observados e esperados de cada hospital pela taxa de mortalidade observada para o conjunto da amostra 15 . Os intervalos de confiança para a razão entre os óbitos observados e esperados e para a taxa de mortalidade ajustada foram calculados empregando-se metodologia proposta por Hosmer \& Lemeshow 16 .

A comparação das distribuições do tempo de permanência e de idade foram avaliadas por meio da prova estatística de Kruskal-Wallis, enquanto as diferenças entre proporções foram avaliadas pelo teste do qui-quadrado. As análises foram realizadas empregando-se os programas Epi-Info 6.04 e Stata.

\section{Resultados}

Em 1999 foram emitidas 25.928 AIH para os hospitais da AP2.2, com uma distribuição de $46,8 \%$ para o sexo masculino e $53,2 \%$ para o feminino, média de idade de 43 anos e desvio padrão de 23 anos. A distribuição do total de AIH pelos hospitais analisados foi de $57,6 \%$ para a Unidade $3,28,3 \%$ para a Unidade $1,13,0 \%$ para a Unidade 2 e 1,2\% para a Unidade 4 . Em relação ao total de hospitalizações, as internações em idosos (maiores de sessenta anos de idade), apresentaram uma distribuição de 28,4\%, 24,5\% e $29,3 \%$, para a Unidade 1, Unidade 2 e Unidade 3 , respectivamente. Todas as internações na Unidade 4 foram de pacientes idosos (Tabela 1). Para a variável tempo de permanência, as unidades apresentaram os seguintes valores de mediana (1o quartil, 3o quartil): Unidade 4: 20 dias (10, 37); Unidade 1: 8 dias $(3,17)$; Unidade 2: 6 dias $(2,14)$ e Unidade 3: 6 dias $(2,13)$. Observando-se que, na Unidade 4 , em todos os quartis de distribuição, os valores foram sempre superiores aos verificados nas demais unidades $(\mathrm{p}=0,000)$.

Na Tabela 2 são apresentadas as distribuições das internações em idosos em relação ao sexo em cada unidade, sendo observada uma diferença significativa ( $\mathrm{p}=0,000)$ para esta variável, com o predomínio do sexo masculino nos hospitais universitários e feminino nos demais. Em relação à distribuição etária, pode ser verificado que, em geral, há um predomínio de internações em idosos mais jovens e um declínio com o aumento da idade. Observa-se, entretanto, diferença significativa $(p=0,000)$ entre faixa etária e unidade de saúde, dada pela maior proporção de idosos acima de oitenta anos, verificada na Unidade 4. A Unidade 1 apresenta internações em idosos mais velhos se comparada aos hospitais universitários, sendo a Unidade 4 responsável pelo aumento da

Tabela 1

Total e proporção de hospitalizações em idosos segundo unidade.

Rio de Janeiro, Brasil, 1999

\begin{tabular}{|c|c|c|c|c|}
\hline \multirow[t]{2}{*}{ Unidade } & \multirow{2}{*}{$\begin{array}{c}\text { Total de } \\
\text { hospitalizações }\end{array}$} & \multicolumn{3}{|c|}{ Hospitalização de idosos } \\
\hline & & $\mathrm{n}$ & $\%$ & IC95\% \\
\hline 1 & 7.333 & 2.083 & 28,4 & $27,4-29,5$ \\
\hline 2 & 3.361 & 822 & 24,5 & $23,0-25,9$ \\
\hline 3 & 14.932 & 4.377 & 29,3 & $28,6-30,1$ \\
\hline 4 & 302 & 302 & 100,0 & - \\
\hline Geral & 25.928 & 7.584 & 29,3 & $28,7-29,8$ \\
\hline
\end{tabular}


Perfil das hospitalizações segundo características do idoso e da hospitalização. Rio de Janeiro, Brasil. 1999 ( $n=7.584)$.

\begin{tabular}{|c|c|c|c|c|c|c|c|c|c|c|}
\hline \multirow[t]{3}{*}{ Características } & \multicolumn{10}{|c|}{ Unidade } \\
\hline & \multicolumn{2}{|c|}{1} & \multicolumn{2}{|c|}{2} & \multicolumn{2}{|c|}{3} & \multicolumn{2}{|c|}{4} & \multicolumn{2}{|c|}{ Geral } \\
\hline & $\mathrm{n}$ & $\%$ & $\mathrm{n}$ & $\%$ & $\mathrm{n}$ & $\%$ & $\mathrm{n}$ & $\%$ & $\mathrm{n}$ & $\%$ \\
\hline \multicolumn{11}{|l|}{ Sexo } \\
\hline Masculino & 1.001 & 48,1 & 437 & 53,2 & 2.335 & 53,3 & 128 & 42,4 & 3.901 & 51,4 \\
\hline Feminino & 1.082 & 51,9 & 385 & 46,8 & 2.042 & 46,7 & 174 & 57,6 & 3.683 & 48,6 \\
\hline \multicolumn{11}{|l|}{ Faixa etária (em anos) } \\
\hline $60-64$ & 435 & 20,9 & 198 & 24,1 & 979 & 22,4 & 25 & 8,3 & 1.637 & 21,6 \\
\hline $65-69$ & 495 & 23,8 & 184 & 22,4 & 1.045 & 23,9 & 46 & 15,2 & 1.770 & 23,3 \\
\hline $70-74$ & 432 & 20,7 & 181 & 22,0 & 1.053 & 24,1 & 62 & 20,5 & 1.728 & 22,8 \\
\hline $75-79$ & 303 & 14,5 & 126 & 15,3 & 687 & 15,7 & 48 & 15,9 & 1.164 & 15,3 \\
\hline $80-84$ & 232 & 11,1 & 76 & 9,2 & 377 & 8,6 & 52 & 17,2 & 737 & 9,7 \\
\hline $85 e+$ & 186 & 8,9 & 57 & 6,9 & 236 & 5,4 & 69 & 22,8 & 548 & 7,2 \\
\hline \multicolumn{11}{|l|}{ Especialidade } \\
\hline Cirurgia & 829 & 39,8 & 564 & 68,6 & 2.830 & 64,7 & - & - & 4.223 & 55,7 \\
\hline Clínica médica & 1.254 & 60,2 & 257 & 31,3 & 1.536 & 35,1 & 302 & 100,0 & 3.349 & 44,2 \\
\hline Psiquiatria & - & - & - & - & 11 & 0,3 & - & - & 11 & 0,1 \\
\hline Tisiologia & - & - & 1 & 0,1 & - & - & - & - & 1 & 0,0 \\
\hline \multicolumn{11}{|l|}{ Capítulos do CID-10 } \\
\hline IX - Doenças do aparelho circulatório & 657 & 31,5 & 84 & 10,2 & 1.142 & 26,1 & 133 & 44,0 & 2.016 & 26,6 \\
\hline VII - Doenças do olho e anexos & 28 & 1,3 & 107 & 13,0 & 849 & 19,4 & - & - & 984 & 13,0 \\
\hline$X I$ - Doenças do aparelho digestivo & 300 & 14,4 & 148 & 18,0 & 465 & 10,6 & 9 & 2,9 & 922 & 12,2 \\
\hline XIV - Doenças do aparelho geniturinário & 126 & 6,1 & 155 & 18,9 & 518 & 11,8 & 12 & 3,9 & 811 & 10,7 \\
\hline II - Neoplasias (tumores) & 176 & 8,5 & 136 & 16,6 & 471 & 10,8 & 11 & 3,6 & 794 & 10,5 \\
\hline$X$ - Doenças do aparelho respiratório & 214 & 10,3 & 52 & 6,3 & 234 & 5,4 & 67 & 22,2 & 567 & 7,5 \\
\hline IV - Doenças endócrinas nutricionais e metabólicas & 179 & 8,6 & 20 & 2,4 & 73 & 1,7 & 28 & 9,3 & 300 & 4,0 \\
\hline $\begin{array}{l}\text { XIX - Lesões, envenenamentos e algumas } \\
\text { outras conseqüências de causas externas }\end{array}$ & 171 & 8,2 & - & - & 104 & 2,4 & - & - & 275 & 3,6 \\
\hline $\begin{array}{l}\text { XIII - Doenças do sistema osteomuscular } \\
\text { e do tecido conjuntivo }\end{array}$ & 32 & 1,5 & 61 & 7,4 & 97 & 2,2 & 1 & 0,3 & 191 & 2,5 \\
\hline I- Algumas doenças infecciosas e parasitárias & 45 & 2,2 & 19 & 2,3 & 94 & 2,1 & 10 & 3,3 & 168 & 2,2 \\
\hline $\mathrm{VI}$ - Doenças do sistema nervoso & 23 & 1,1 & 13 & 1,6 & 80 & 1,8 & 8 & 2,7 & 124 & 1,6 \\
\hline $\begin{array}{l}\text { XVIII - Sintomas, sinais e achados anormais } \\
\text { de exames clínicos e de laboratório não } \\
\text { classificados em outra parte }\end{array}$ & 57 & 2,7 & 12 & 1,5 & 43 & 0,9 & - & - & 112 & 1,5 \\
\hline $\begin{array}{l}X X I \text { - Fatores que influenciam o estado de saúde } \\
\text { e o contato com os serviços de saúde }\end{array}$ & 15 & 0,7 & - & - & 92 & 2,1 & - & - & 107 & 1,4 \\
\hline XII - Doenças da pele e do tecido subcutâneo & 32 & 1,5 & 8 & 0,9 & 36 & 0,8 & 8 & 2,7 & 84 & 1,1 \\
\hline $\begin{array}{l}\text { III - Doenças do sangue e dos órgãos } \\
\text { hematopoéticos e alguns transtornos imunitários }\end{array}$ & 20 & 0,9 & 5 & 0,6 & 29 & 0,7 & 4 & 1,3 & 58 & 0,8 \\
\hline V - Transtornos mentais e comportamentais & 2 & 0,1 & - & - & 13 & 0,3 & 11 & 3,6 & 26 & 0,3 \\
\hline XX - Causas externas de morbidade e de mortalidade & 2 & 0,1 & - & - & 22 & 0,5 & - & - & 24 & 0,3 \\
\hline VIII - Doenças do ouvido e da apófise mastóide & 1 & 0,05 & 2 & 0,2 & 10 & 0,2 & - & - & 13 & 0,2 \\
\hline $\begin{array}{l}\text { XVII - Malformações congênitas, deformidades } \\
\text { e anomalias cromossômicas }\end{array}$ & 3 & 0,14 & - & - & 4 & 0,1 & - & - & 7 & 0,1 \\
\hline XV - Gravidez parto e puerpério & 0 & 0,0 & - & - & 1 & 0,02 & - & - & 1 & 0,01 \\
\hline
\end{tabular}


média de idade de internação dos idosos da AP2.2, ou seja, neste hospital internam os idosos com idades extremas.

Para as especialidades de internação, a distribuição de AIH se dá em grande parte pela Cirurgia (55,7\%) e Clínica Médica (44,2\%), sendo constatados neste ano, 11 casos de internação para Psiquiatria na Unidade 3 e apenas um caso para Tisiologia, na Unidade 2 (Tabela 2).

Do total de internações analisadas apenas em 19,5\% (1.481) havia descrito o diagnóstico secundário. O motivo da internação foi analisado com base no diagnóstico principal relatado nas AIH, que foram agrupados segundo os capítulos da CID-10, sendo obtida a seguinte distribuição: doenças do aparelho circulatório (26,6\%); doenças do olho e anexos (12,8\%); doenças do aparelho digestivo (12,2\%); doenças do aparelho geniturinário ( $10,7 \%)$; neoplasias $(10,1 \%)$ e doenças do aparelho respiratório $(7,5 \%)$ (Tabela 2). Embora as doenças do aparelho cardiovascular tenham sido o motivo de internação mais freqüente em todas as unidades, estas diferiram significativamente em relação às distribuições dos motivos da internação ( $\mathrm{p}=0,000)$, sendo, por exemplo, observada uma maior proporção de hospitalizações por doenças do aparelho respiratório nos hospitais não universitários e uma proporção bem mais elevada de hospitalizações por doenças do olho e anexos nos hospitais universitários (Tabela 2). Já os diagnósticos principais mais freqüentes foram distribuídos da seguinte maneira: catarata senil incipiente $(7,8 \%)$; hiperplasia de próstata $(4,7 \%)$; insuficiência cardíaca congestiva $(2,9 \%)$ e bloqueio atriventricular total $(2,8 \%)$.

Na Tabela 3 são apresentadas as proporções de óbito segundo características do idoso e da hospitalização. Não foram observadas diferenças significativas na mortalidade hospitalar segundo sexo $(\mathrm{p}=0,120)$. Quanto à distribuição segundo faixa etária foi verificado um crescimento significativo da mortalidade com o envelhecimento, que é mais expressivo a partir dos 75 anos de idade $(p=0,000)$. Com relação ao motivo da internação, as doenças infecciosas e parasitárias, as doenças do aparelho respiratório, os transtornos mentais e comportamentais, as doenças endócrinas nutricionais e metabólicas, as doenças do aparelho circulatório e as neoplasias foram os capítulos da CID-10 que obtiveram as maiores taxas de mortalidade.

A Unidade 4 foi o hospital com a maior taxa de mortalidade bruta encontrada, sendo seguida pela Unidade 1. Estes hospitais apresentaram taxas brutas de mortalidade bem superiores às verificadas para os hospitais universitários (Tabela 3). Após o ajuste para diferenças no perfil dos pacientes internados segundo idade e motivo da internação, verifica-se uma diminuição importante das taxas das Unidades 4 e 1, que passaram a apresentar valores de taxas ajustadas semelhantes. Já os hospitais universitários apresentaram taxas ajustadas ligeiramente superiores às taxas de mortalidade brutas. Sendo assim, com o ajuste observou-se uma redução do diferencial da mortalidade entre os hospitais, entretanto, os hospitais universitários permaneceram apresentando taxas significativamente menores que as verificadas para as demais unidades (Figura 1).

\section{Discussão}

Neste estudo observamos que durante o período considerado as internações em idosos representaram aproximadamente um terço do total das internações nos hospitais da AP2.2, superando o porcentual de $18,7 \%$ observado em 1997 por Costa et al. 17, em um estudo sobre mortalidade e internação hospitalar na população idosa brasileira. Esta diferença pode ser explicada pelo elevado porcentual de idosos na população da AP2.2, que em 1991 era igual a $17,0 \%$, sendo maior que a proporção de idosos verificada para o conjunto da cidade do Rio de Janeiro (11,0\%) 18. Em concordância com outros trabalhos 3,4 , observamos que as hospitalizações em idosos estiveram associadas a um prolongado tempo de permanência hospitalar, especialmente na Unidade 4.

O idoso, proporcionalmente, tende a apresentar mais episódios de doenças, em geral crônicas, levando ao aumento nos gastos em saúde, já que o custo com o idoso tende a ser maior do que para as outras faixas etárias 3,5,17.

Costa et al. 17 encontraram como principais capítulos de internação, para os três anos de análise (1995, 1996 e 1997), as doenças do aparelho circulatório, doenças do aparelho respiratório e doenças do aparelho digestivo. Nos idosos da AP2.2, as principais causas de internação foram as doenças do aparelho circulatório, doenças do olho e anexos, doenças do aparelho digestivo, doenças do aparelho geniturinário, neoplasias e doenças do aparelho respiratório. Além de diferenças na prevalência de doenças nas populações estudadas, outro fator que pode explicar os resultados divergentes obtidos nos dois estudos é o perfil das unidades analisadas. Em nosso estudo, das quatro unidades estudadas duas eram hospitais universitários. A análise dos dados com maior detalhamento mostrou que catarata senil incipiente; hiperplasia de próstata; insuficiência 
Mortalidade hospitalar segundo características do idoso e da hospitalização. Rio de Janeiro, Brasil, 1999.

\begin{tabular}{|c|c|c|c|c|}
\hline \multirow[t]{2}{*}{ Características } & \multirow[t]{2}{*}{ Total } & \multicolumn{3}{|c|}{ Mortalidade } \\
\hline & & $\mathrm{n}$ & $\%$ & IC95\% \\
\hline \multicolumn{5}{|l|}{ Sexo } \\
\hline Masculino & 3.901 & 490 & 12,6 & $11,5-13,6$ \\
\hline Feminino & 3.683 & 507 & 13,8 & $12,7-14,9$ \\
\hline \multicolumn{5}{|l|}{ Faixa etária (em anos) } \\
\hline $60-64$ & 1.637 & 168 & 10,3 & $8,8-11,8$ \\
\hline $65-69$ & 1.770 & 189 & 10,7 & $9,3-12,2$ \\
\hline $70-74$ & 1.728 & 198 & 11,5 & $9,9-13,1$ \\
\hline $75-79$ & 1.164 & 180 & 15,5 & $13,4-17,7$ \\
\hline $80-84$ & 737 & 132 & 17,9 & $15,2-20,9$ \\
\hline 85 e + & 548 & 130 & 23,7 & $20,2-27,5$ \\
\hline \multicolumn{5}{|l|}{ Capítulo } \\
\hline I- Algumas doenças infecciosas e parasitárias & 168 & 78 & 46,4 & $38,7-54,3$ \\
\hline$X$ - Doenças do aparelho respiratório & 567 & 173 & 30,5 & $26,7-34,5$ \\
\hline V - Transtornos mentais e comportamentais & 26 & 6 & 23,1 & $8,9-46,4$ \\
\hline IV - Doenças endócrinas nutricionais e metabólicas & 300 & 63 & 21,0 & $16,5-26,1$ \\
\hline IX - Doenças do aparelho circulatório & 2.016 & 362 & 17,9 & $16,3-19,7$ \\
\hline II - Neoplasias (tumores) & 794 & 134 & 16,9 & $14,3-19,7$ \\
\hline $\begin{array}{l}\text { XVIII - Sintomas, sinais e achados anormais de exames } \\
\text { clínicos e de laboratório não classificados em outra parte }\end{array}$ & 112 & 19 & 16,9 & $10,5-25,2$ \\
\hline $\mathrm{VI}$ - Doenças do sistema nervoso & 124 & 19 & 15,3 & $9,5-22,9$ \\
\hline $\begin{array}{l}\text { III - Doenças do sangue e dos órgãos hematopoéticos } \\
\text { e alguns transtornos imunitários }\end{array}$ & 58 & 8 & 13,8 & $6,1-25,4$ \\
\hline XII - Doenças da pele e do tecido subcutâneo & 84 & 9 & 10,1 & $5,0-19,4$ \\
\hline $\begin{array}{l}\text { XIX - Lesões, envenenamentos e algumas outras } \\
\text { conseqüências de causas externas }\end{array}$ & 275 & 24 & 8,7 & $5,7-12,7$ \\
\hline$X I$ - Doenças do aparelho digestivo & 922 & 66 & 7,2 & $5,6-9,0$ \\
\hline XIV - Doenças do aparelho geniturinário & 811 & 30 & 3,7 & $2,5-5,2$ \\
\hline XIII - Doenças do sistema osteomuscular e do tecido conjuntivo & 191 & 6 & 3,1 & $1,2-6,7$ \\
\hline VII - Doenças do olho e anexos & 984 & 0 & 0,0 & $0,0-0,4$ \\
\hline $\begin{array}{l}\text { XXI - Fatores que influenciam o estado de saúde } \\
\text { e o contato com os serviços de saúde }\end{array}$ & 107 & 0 & 0,0 & $0,0-3,3$ \\
\hline XX - Causas externas de morbidade e de mortalidade & 24 & 0 & 0,0 & $0,0-14,2$ \\
\hline VIII - Doenças do ouvido e apófise mastóide & 13 & 0 & 0,0 & $0,0-24,7$ \\
\hline $\begin{array}{l}\text { XVII - Malformações congênitas, deformidades } \\
\text { e anomalias cromossômicas }\end{array}$ & 7 & 0 & 0,0 & $0,0-41,0$ \\
\hline XV - Gravidez parto e puerpério & 1 & 0 & 0,0 & $0,0-97,5$ \\
\hline \multicolumn{5}{|l|}{ Unidade } \\
\hline $1^{*}$ & 2.083 & 530 & 25,4 & $23,6-27,4$ \\
\hline $2^{\star \star}$ & 822 & 68 & 8,3 & $6,5-10,4$ \\
\hline $3^{\star \star \star}$ & 4.377 & 285 & 6,5 & $5,8-7,3$ \\
\hline $4^{\star \star \star \star}$ & 302 & 114 & 37,7 & $32,3-43,5$ \\
\hline
\end{tabular}

* Hospital Municipal com atendimento de emergência; ** Hospital Universitário sem emergência;

*** Hospital Universitário sem emergência; **** Hospital Municipal com atendimento especificamente Geriátrico. 
Taxas de mortalidade hospitalar bruta e ajustada* e respectivos intervalos de confiança, segundo unidade de saúde. Rio de Janeiro, Brasil, 1999.

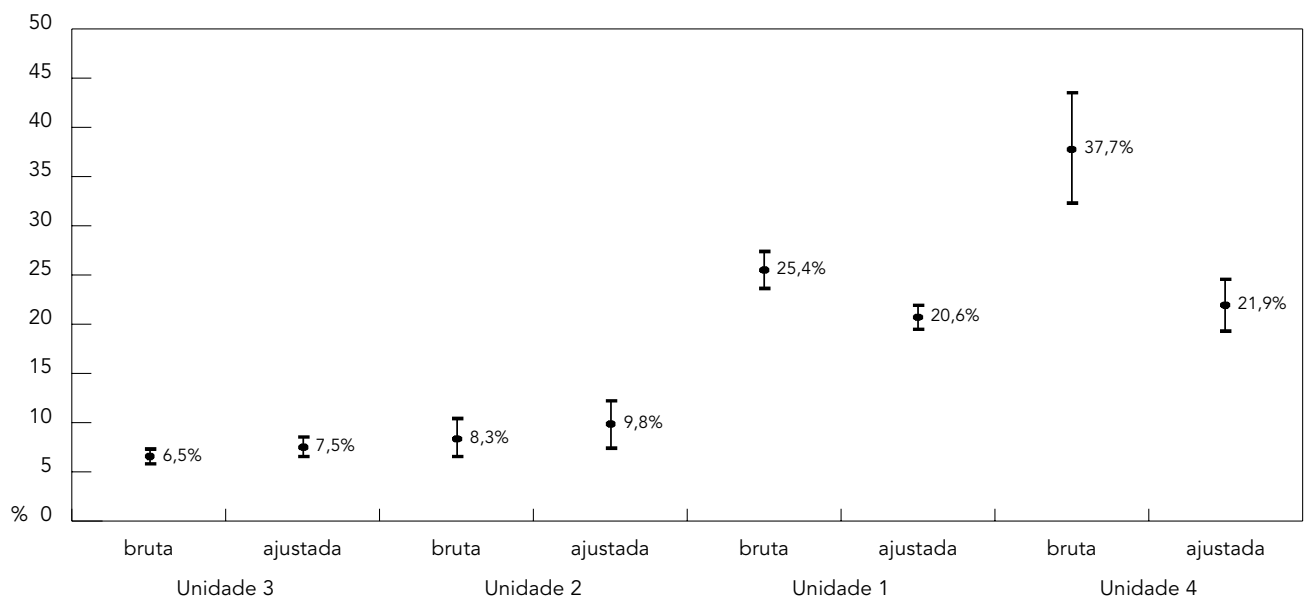

* Ajustada para idade e motivo de hospitalização.

cardíaca congestiva e bloqueio atriventricular total foram os diagnósticos primários mais freqüentes entre os hospitais da AP2.2. As doenças do olho e anexos obtiveram o segundo lugar dentre as causas de internação, este fato pode explicar a grande proporção de hospitalizações observadas, especialmente nos hospitais universitários, na especialidade de cirurgia.

Este trabalho utiliza informações existentes nos grandes bancos de dados administrativos nacionais sobre internações hospitalares. A utilização desta e de outras bases hoje disponíveis em nosso meio em estudos epidemiológicos apresenta vantagens como: o baixo custo para a condução dos estudos, a possibilidade de inferência para o país e comparações ao longo do tempo. Por outro lado, estudos deste tipo são limitados às informações disponíveis e à qualidade destas informações, destacando-se como desvantagens: a subenumeração de óbitos e a grande proporção de óbitos por causas mal definidas 17. Considerando estas limitações, é importante, entretanto, ressaltar o potencial destas fontes de dados para a condução de estudos exploratórios, que podem servir como um ponto de partida para a realização de análises mais detalhadas com base na utilização de outras fontes de dados (por exemplo, revisão de prontuários médicos e entrevistas).

Com relação aos bancos de dados administrativos hospitalares, a Agency for Healthcare
Research and Quality 11 vem propondo a utilização de um conjunto de indicadores construídos a partir desses bancos que permitiriam o monitoramento do cuidado ambulatorial (por meio de um conjunto de condições cujas hospitalizações poderiam ser prevenidas pelo cuidado ambulatorial de qualidade) e do cuidado hospitalar (incluindo a mortalidade hospitalar, a freqüência e a adequação do uso de determinados procedimentos e a ocorrência de complicações durante a internação) 11 .

Neste estudo buscamos avaliar comparativamente a mortalidade hospitalar nas unidades estudadas, levando em consideração que as mesmas possuem diferentes características (hospitais municipais ou universitários, com ou sem emergência, e, ainda um hospital especializado em tratamento geriátrico) e atendem a perfis de casos distintos. Tanto o motivo da internação como a idade se mostraram associados à mortalidade hospitalar e ao mesmo tempo apresentaram uma distribuição desigual entre as unidades estudadas. Os hospitais universitários apresentaram uma clientela mais jovem e uma proporção elevada de hospitalizações para correção cirúrgica de catarata senil, dois fatores associados a uma menor mortalidade hospitalar. A Unidade 1, provavelmente por ser o único hospital de emergência, interne pacientes mais graves, e a Unidade 4 atende a uma clientela de pacientes crônicos e mais 
idosos, ou seja, pacientes mais graves, requerendo cuidados prolongados e que, portanto, tende a aumentar as taxas de mortalidade.

Sendo assim, estes diferentes perfis de clientela podem explicar, ainda que parcialmente, as diferenças nas taxas brutas de mortalidade nas unidades estudadas. Quando estas taxas foram ajustadas, percebemos que a Unidade 4 e a Unidade 1 obtiveram taxas bastante semelhantes entre si, embora continuassem distantes dos hospitais universitários, estes com um porcentual de mortalidade bruto menor, em ambos, do que o ajustado. Cabe ressaltar, entretanto, que o ajuste por nós realizado não considerou outros aspectos relacionados à gravidade dos casos, como a presença de instabilidade clínica e de comorbidades.

Com relação a este último aspecto, uma série de medidas de ajuste de risco baseadas na informação sobre comorbidades presentes no momento da internação, vem sendo proposta para a análise comparativa de desempenho de hospitais que empregam os bancos administrativos 19. O banco de dados das AIH apresentam apenas um campo para o preenchimento de diagnóstico secundário, o que dificulta o cálculo das medidas de ajuste de risco para comorbidades. Martins et al. 9, aplicando o índice de comorbidade de Charlson em um estudo empregando as bases do SIH/SUS, encontraram um baixo poder para a discriminação da gravidade dos casos, o que os autores atribuíram à baixa qualidade da informação diagnóstica na base do SIH/SUS. Esses autores observaram que em apenas $18,6 \%$ das internações estudadas havia anotações sobre o diagnóstico secundário, resultado semelhante ao por nós observado.

Outro fator a ser considerado diz respeito ao método de ajuste de taxas empregado. Este baseia-se no cálculo de uma razão entre óbitos observados e esperados, sendo estes últimos estimados com base em um modelo ajustado para o conjunto das hospitalizações. A razão entre óbitos observados e esperados pode ser interpretada como uma razão de mortalidade padronizada (padronização indireta), que ao ser multiplicada pela taxa de mortalidade para o conjunto das hospitalizações (taxa de mortalidade hospitalar média) fornece a taxa de mortalidade ajustada hospitalar 20. Shwartz et al. 15 chamam a atenção para o fato desta metodologia ser limitada para a realização da comparação da performance de unidades de saúde nas situações onde estas atendam a perfis de clientela muito distintos, como foi o caso das unidades aqui estudadas. Este problema poderia ser minimizado pela comparação do resultado relativo a alguns diagnósticos específicos, que foram motivo de hospitalização freqüente em todas as unidades estudadas e que já foram demostrados como indicadores sensíveis da qualidade do cuidado (por exemplo, a insuficiência cardíaca congestiva) 11 . Neste caso, entretanto, seria necessário aumentar o período estudado de forma a garantir um número suficiente de observações em todas as unidades, visando a minimizar o efeito da variação aleatória nas estimativas obtidas 15,20 . Outra alternativa seria a aplicação de modelos multiníveis, sendo oportuno neste caso aumentar o número de unidades hospitalares avaliadas 20.

Por fim, outros fatores que devem ser considerados na interpretação dos resultados são a possível ocorrência de sub-registro de formulário de autorização de internação hospitalar em relação ao total de hospitalizações realizadas nas unidades de saúde e práticas diferenciadas em relação a alta de pacientes. O sub-registro poderia levar ao enviesamento dos resultados, especialmente se o mesmo ocorresse de forma diferente entre casos que evoluíram ou não para o óbito. Já a alta precoce de pacientes poderia levar a subestimativa da mortalidade associada ao episódio de hospitalização. Este último problema poderia ser minimizado pela análise da mortalidade em trinta dias, que pode ser calculada a partir do relacionamento dos bancos dos formulários AIH com as bases de mortalidade, empregando-se o método de relacionamento probabilístico de registros 21 .

Apesar das limitações da análise acima apontadas, o grande diferencial da mortalidade observada entre os hospitais universitários e não universitários sugere a necessidade da realização de uma avaliação mais detalhada nesses últimos. Poderia ser realizada a revisão de prontuários de uma amostra de hospitalizações de alguns diagnósticos específicos (por exemplo, insuficiência cardíaca congestiva), visando a avaliar se características clínicas dos pacientes hospitalizados e ligadas ao processo de cuidado poderiam explicar os resultados observados.

Concluindo, os bancos dos formulários AIH podem ser úteis não somente para a descrição do perfil de hospitalização em idosos, como também para avaliações exploratórias de resultados da performance hospitalar. O emprego destas fontes e da metodologia de ajuste de risco pode fornecer informações úteis para o planejamento e execução de avaliações mais detalhadas da qualidade da assistência hospitalar. O aumento do número de campos disponíveis para o registro de diagnósticos secundários, a criação de campos indicadores visando 
a distinção entre comorbidades e complicações desenvolvidas durante a hospitalização e o preenchimento de formulários AIH para todas as hospitalizações, representam medidas que permitiram uma melhor utilização destas fontes de dados para fins de avaliação.

\section{Resumo}

Os objetivos deste estudo são analisar o perfil de morbi-mortalidade em idosos hospitalizados em dois hospitais universitários e dois não universitários, da Área de planejamento 2.2 da cidade do Rio de Janeiro, Brasil, no ano de 1999, comparando as taxas de mortalidade hospitalar, ajustando para diferenças no perfil. Os dados foram obtidos do Sistema de Informações Hospitalares do Sistema Único de Saúde (SIH/SUS). O modelo logístico foi ajustado incluindo as variáveis idade e diagnóstico primário, utilizado para calcular as taxas de mortalidade hospitalar ajustadas. As internações hospitalares em idosos $(n=7.584)$ representaram 29,3\% do total de 25.928 internações realizadas nessas unidades. Catarata senil $(7,8 \%)$ foi a causa mais freqüente, seguida de hiperplasia de próstata (4,7\%), insuficiência cardiaca congestiva $(2,9 \%)$ e bloqueio atrioventricular total (2,8\%). Os hospitais não universitários apresentaram taxas de mortalidade hospitalar maiores do que as dos hospitais universitários, mesmo depois do ajuste para diferenças no perfil de casos em relação à idade e diagnóstico principal. $O$ uso dos bancos de dados do SIH/SUS e da metodologia de ajuste de risco representam uma alternativa para avaliações exploratórias de resultados de cuidados de saúde.

Saúde do Idoso; Hospitalização; Mortalidade Hospitalar; Morbidade

\section{Colaboradores}

A. C. S. Amaral e C. M. Coeli participaram na coleta de dados, análise, redação e revisão de texto. A. C. S. Amaral, M. C. E. Costa e V. S. Cardoso contribuíram na fase de planejamento e coleta de dados. Todos os autores colaboraram no planejamento da pesquisa.

\section{Agradecimentos}

Às residentes de Saúde Coletiva Ana Lúcia Araújo de Toledo e Carla Rodrigues Fernandes que contribuíram na fase de coleta de dados. 


\section{Referências}

1. Ramos LR, Veras RP, Kalache A. Envelhecimento populacional: uma realidade brasileira. Rev Saúde Pública 1987; 21:211-24.

2. World Health Organization. Population ageing: a public health challenge. Geneva: World Health Organization Press Office; 1998. (Fact Sheet 135).

3. Cavalcanti MGPH, Saad PM. Os idosos no contexto da Saúde Pública. In: Fundação Sistema Estadual de Análise de Dados, organizador. O idoso na Grande São Paulo, São Paulo: Fundação Sistema Estadual de Análise de Dados; 1990. p. 181-205.

4. Veras RP, Coutinho E, Coeli CM. Transtornos mentais em idosos: a contribuição da epidemiologia. In: Veras RP, organizador. Terceira idade: desafios para o terceiro milênio. Rio de Janeiro: Relume-Dumará/Universidade Aberta da Terceira Idade/Universidade do Estado do Rio de Janeiro; 1997. p. 15-40.

5. Chaimowicz F. A saúde dos idosos brasileiros às vésperas do século XXI: problemas, projeções e alternativas. Rev Saúde Pública 1997; 31:184-200.

6. Veras RP, Ramos LR, Kalache A. Crescimento da população idosa no Brasil: transformações e conseqüências na sociedade. Rev Saúde Pública 1987; 21:225-33.

7. Telarolli RJ, Machado JCMS, Carvalho F. Perfil demográfico e condições sanitárias dos idosos em área urbana do Sudeste do Brasil. Rev Saúde Pública 1996; 30:485-98.

8. Gouvêa CSD, Travassos C, Fernandes C. Produção de serviços e qualidade da assistência hospitalar no Estado do Rio de Janeiro, Brasil - 1992 a 1995. Rev Saúde Pública 1997; 31:601-17.

9. Martins M, Travassos C, Noronha JC. Sistema de Informações Hospitalares como ajuste de risco em índices de desempenho. Rev Saúde Pública 2001; 35:185-92.

10. Garnick DW, Hendricks AM, Comstock CB. Measuring quality of care: fundamental information from administrative datasets. Int J Qual Health Care 1994; 6:163-77.

11. Agency for Healthcare Research and Quality. AHRQ Quality Indicators - guide to inpatient quality indicators: quality of care in hospitals - volume, mortality and utilization. Rockville: Agency for Healthcare Research and Quality; 2002. (AHRQ Pub. no 02-RO204).
12. Guerra HL, Barreto SM, Uchôa E, Firmo JOA, Lima-Costa MF. A morte de idosos na Clínica Santa Genoveva, Rio de Janeiro: um excesso de mortalidade que o sistema público de saúde poderia ter evitado. Cad Saúde Pública 2000; 16:545-51.

13. Organização Mundial da Saúde. Classificação Estatística Internacional de Doenças e Problemas Relacionados à Saúde, 10a Revisão. v. 1. São Paulo: Centro Colaborador da OMS para a Classificação de Doenças em Português; 1995.

14. Hosmer DW, Lesmeshow S. Applied logistic regression. 2nd Ed. New York: John Wiley \& Sons; 2000.

15. Scwartz M, Ash AS, Iezzoni LI. Comparing outcomes across providers. In: Ieozzoni LI, editor. Risk adjustment for measuring healthcare outcomes. 2nd Ed. Chicago: Health Administration Press; 1997. p. 471-516.

16. Hosmer DW, Lemeshow S. Confidence interval estimates of an index of quality performance based on logistic regression models. Stat Med 1995; 14:2161-72.

17. Costa MFFL, Guerra HL, Barreto SM, Guimarães RM. Diagnóstico da situação da população idosa brasileira: um estudo da mortalidade e das internações hospitalares públicas. Inf Epidemiol SUS 2000; 9:23-41.

18. Empresa Municipal de Informática e Planejamento. Anuário estatístico da cidade do Rio de Janeiro, 1993/1994. Rio de Janeiro: Empresa Municipal de Informática e Planejamento; 1995.

19. Schneeweiss S, Maclure M. Use of comorbidity scores for control of confounding in studies using administrative databases. Int J Epidemiol 2000; 29:891-8.

20. Goldstein H, Spiegelhalter DJ. League tables and their limitations: statistical issues in comparisons of institutional performance. J R Statist Soc (A) 1996; 159:385-443.

21. Camargo Jr. KR, Coeli CM. Reclink: aplicativo para o relacionamento de banco de dados implementando o método probabilistic record linkage. Cad Saúde Pública 2000; 16:439-47.

Recebido em 20/Mai/2003

Versão final reapresentada em 14/Out/2003

Aprovado em 21/Jun/2004 